Revista Destaques Acadêmicos, Lajeado, v. 9, n. 3, 2017. ISSN 2176-3070 DOI: http://dx.doi.org/10.22410/issn.2176-3070.v9i3a2017.1511 www.univates.br/revistas

\title{
O CUIDAR EM ONCOLOGIA PEDIÁTRICA: UM ESTUDO BASEADO NO PROCESSO DE ENFERMAGEM
}

\author{
Mary Elizabeth de Santana ${ }^{1}$, Elanny Glicia Oliveira da Costa ${ }^{2}$, \\ Anderson Roberto de Sales Corrêa ${ }^{3}$, Wagner Luiz Oliveira Ximenes ${ }^{4}$
}

Resumo: Introdução: A assistência de enfermagem em oncologia desenvolve-se
pelos cuidados preventivos, curativos e paliativos. Os cuidados curativos buscarão
diminuição dos efeitos imediatos e tardios do tratamento quimioterápico e prevenção
de complicações evitáveis da patologia oncológica por meio da Sistematização da
Assistência de Enfermagem. Este estudo tem como objetivo descrever os diagnósticos
de enfermagem e cuidados prestados ao paciente com câncer infanto-juvenil. Métodos:
Trata-se de um estudo retrospectivo, com abordagem quantitativa, realizado a partir de
registros de prontuário no departamento de arquivo médico de um CACON. De crianças
e adolescentes internados na clínica pediátrica no período de 2011 e 2012 . A pesquisa
possui parecer no524.165 CEP/UFPA. Resultados: O estudo foi evidenciou 292 pacientes
com câncer entre 2011 e 2012 . A população em oncopediatria foi em sua maioria do sexo
masculino (59,59\%), e a neoplasia mais evidente foi a Leucemia Linfoblástica Aguda.
Nesta pesquisa foi identificado 584 diagnósticos de enfermagem, com uma média de
2,01 diagnósticos de enfermagem por paciente. Os principais títulos diagnósticos foram:
Risco para infecção (30,14\%); Conforto prejudicado (15,07\%); Nutrição desequilibrada
menos que as necessidades corporais com (11,82\%) e Mobilidade física prejudicada
com (6,51\%). Foi possível visualizar a grande carga de trabalho despendida ao paciente
oncohematológico em pediatria, e realização do processo de enfermagem de modo
eficaz na instituição. Conclusão: A sistematização da assistência mostrou-se presente

1 Graduada em Enfermagem Pós-Doutorado em Enfermagem Fundamental (Universidade de São Paulo, USP, Brasil - 2015). Professora adjunta de fundamentos de enfermagem da Universidade Federal do Pará.

2 Graduação em Licenciatura e Bacharelado em Enfermagem. Mestranda em Saúde Animal na Amazônia (Universidade Federal do Pará, UFPA, 2017).

3 Graduação em Licenciatura e Bacharelado em Enfermagem (Universidade Federal do Pará, UFPA, 2014). Residente em enfermagem oncológica pela Universidade do Estado do Pará.

4 Graduação em Medicina pela Universidade do Estado do Pará. Afiliado a Universidade do Estado do Pará. 
em todos os registros de prontuário da internação, seja por título diagnóstico ou por prestação de cuidados de enfermagem.

Palavras-chave: Oncologia. Pediatria. Cuidados de Enfermagem.

\section{INTRODUÇÃO}

As neoplasias nas crianças e adolescentes correspondem a cerca de $0,5 \%$ a 3\% de todas os cânceres, na maioria das populações. Sendo responsável por uma das principais causas de óbito na população pediátrica. O câncer infantojuvenil atinge principalmente células do sistema hematopoiético e os tecidos de sustentação, e na maioria são de origem embrionária, enquanto que, no adulto, afetas as células do epitélio que recobrem os diferentes órgãos (LINS, 2011).

Em relação ao tratamento, os efeitos adversos, o planejamento e implementação dos cuidados de enfermagem para os pacientes e suas respectivas famílias, devem ser repassados integralmente e de forma simples, pois os pacientes informados aderem com mais facilidade ao tratamento e são menos suscetíveis às complicações relacionadas ao autocuidado, tornando-os, mas satisfeitos com o cuidado que recebem e com as informações adequadas de como se cuidarem (BASTABLE, 2010).

A prática do cuidar em oncologia infanto-juvenil é desafiante, uma vez que pressupõe, além de recursos materiais e terapêuticos específicos, uma equipe de saúde atenta para o que permeia este universo. Há necessidade de profissionais com responsabilidade, compromisso; preparo adequado e sensibilidade para realizar o cuidado direcionado a criança e adolescente, e é neste contexto, que se insere o profissional enfermeiro, cuja produção do cuidado qualificado é influenciada pelo conforto com a realidade do câncer infantil, que inclui aspectos práticos e emocionais (AMADOR et al., 2011).

O cuidado direcionado a criança e adolescente com câncer é complexo, pois envolve múltiplos aspectos, e nesse contexto é importante que o enfermeiro articule saberes e práticas, que busque o aperfeiçoamento em oncologia, e tenha habilidades para gerenciar o cuidado de enfermagem, pois este quando realizado considerando as tecnologias em saúde, é capaz de resolver problemas, estreitar vínculos e favorecer a corresponsabilidade nas relações do cuidado (SILVA et al., 2013).

Durante levantamento bibliográfico e de pesquisa no CACON da região norte, foi identificado baixa densidade de estudos produzidos e publicados nesta região com o foco para o processo de enfermagem em oncologia pediátrica. Sendo a maioria voltado para aspectos qualitativos relacionado ao cuidar. A escolha deste tema surgiu a partir da questão: Quais o perfil dos diagnósticos e intervenções de enfermagem implementados em oncologia pediátrica e a sua relação com os sinais e sintomas que crianças e adolescentes com câncer vivenciam. 
Sendo assim será exposto o resultado da pesquisa referente ao objetivo de descrever os diagnósticos e cuidados de enfermagem prestados ao paciente com câncer infanto-juvenil.

\section{MATERIAIS E MÉTODOS}

A pesquisa é baseada em um estudo descritivo, retrospectivo com abordagem quantitativa, a metodologia quantitativa é a descrição objetiva e sistemática do conteúdo analisado que é apresentado com auxílio de quadros, tabelas e medidas que irão traduzir as opiniões e informações analisadas por meio de técnicas estatísticas 5 . O estudo foi realizado no Departamento de Arquivo Médico e Estatístico em um hospital de referência em oncologia da região Norte.

O estudo foi operacionalizado após autorização do Comitê de Ética em Pesquisa com Seres Humanos e Animais da Universidade Federal do Pará sob o parecer consubstanciado de número 524.165 e número do CAEE: 23728313.5.0000.0018. Como fonte de dados foi utilizado prontuários de crianças e adolescentes acometidos por câncer na faixa etária de 0 a 19 anos com diagnóstico de novo caso nos anos de 2011 e 2012, a coleta de dados foi realizada a partir de formulário adaptado do Registro de Câncer de Base Populacional, adicionando-se aspectos relacionados ao cuidado de enfermagem. Os dados foram analisados através de estatística descritiva, realizando cálculos de valores absolutos, porcentagem, desvio padrão e média aritmética dos dados.

\section{RESULTADOS}

A incidência de câncer infanto-juvenil no período estudo teve pouca variação nos dois anos, sendo 174 casos novos para 2011 e 118 para 2012. Em relação ao tipo de câncer, a LLA esteve presente em 38,19\% das crianças entre 0 a 5 anos $(n=55)$ e $27,16 \%$ das crianças entre 6 a 10 anos $(n=22)$, o osteossarcoma foi mais incidente em adolescentes de 11 a 19 anos com 23,88\% (N=16).

O processo de enfermagem utilizado como protocolo na instituição de estudo para os títulos diagnósticos de enfermagem é o "Manual de diagnósticos de enfermagem de Lynda Carpenito". O processo de enfermagem é definido como um método sistemático e dinâmico que estabelece um diagnóstico a partir do estado de saúde, realiza intervenções e em seguida um plano de cuidados com intuito de atingir uma eficácia (QUECHO, 2012).

Durante a pesquisa em prontuários, foi identificado 584 diagnósticos de enfermagem válidos. Com uma média de 2,01 diagnóstico por paciente e desvio padrão de $\pm 2,11$ para cada paciente, com número máximo de 10 diagnósticos e mínimo de nenhum diagnóstico por criança ou adolescente.

Visualizaram-se como os cinco principais diagnósticos de enfermagem: Risco para infeç̧ão, Conforto prejudicado, Nutrição desequilibrada menos que as necessidades corporais, Mobilidade física prejudicada e Risco para lesão. 
Foram encontrados ainda outros diagnósticos de grande significância em oncopediatria, porém em baixa porcentagem, como o Risco para lesão, Integridade da pele prejudicada e Mucosa oral prejudicada todos diretamente relacionados aos efeitos indesejados da poliquimioterapia antineoplásica aos quais os pacientes são submetidos e que os deixam em frequente imunossupressão medular.

Na tabela 1 podemos observar os 15 principais diagnósticos identificado no estudo:

Tabela 1: Principais diagnósticos de enfermagem

\begin{tabular}{l|c|c}
\hline DIAGNÓSTICO DE ENFERMAGEM & N & $\%$ \\
\hline Risco para infecção & 176 & 30,14 \\
Conforto prejudicado & 88 & 15,07 \\
Nutrição Desequilibrada, menos que as necessidades corporais & 69 & 11,82 \\
Mobilidade física prejudicada & 38 & 6,51 \\
Risco para lesão & 22 & 3,77 \\
Dor aguda & 21 & 3,60 \\
Sono e repouso prejudicado & 21 & 3,60 \\
Integridade da pele prejudicada & 17 & 2,91 \\
Integridade tissular prejudicada & 12 & 2,05 \\
Mucosa oral prejudicada & 12 & 2,05 \\
Ansiedade & 10 & 1,71 \\
Deambulação prejudicada & 8 & 1,37 \\
Hipertermia & 6 & 1,03 \\
Dor crônica & 6 & 1,03 \\
Déficit no autocuidado & 6 & 1,03 \\
Outros & 72 & 12,26 \\
\hline
\end{tabular}

Fonte: DAME- Divisão de Arquivo Médico e Estatístico/HOL, 2014.

Em relação as características definidoras, foi realizada nova filtração onde se elencou os 7 diagnósticos de enfermagem considerando a sua frequência no estudo. A partir da análise e desmembramento dos diagnósticos foi possível a construção da tabela 2: 
TABELA 2 - Principais diagnósticos de enfermagem e seus fatores relacionados

\begin{tabular}{|c|c|c|c|}
\hline $\begin{array}{l}\text { Diagnóstico de } \\
\text { Enfermagem }\end{array}$ & FATOR RELACIONADO & $\mathrm{N}$ & $\%$ \\
\hline Risco para & Câncer & 6 & 3,45 \\
\hline \multirow[t]{5}{*}{ Infecção } & Local de invasão do organismo & 77 & 44,25 \\
\hline & Procedimentos invasivos & 6 & 3,45 \\
\hline & Comprometimento das defesas do hospedeiro & 68 & 39,08 \\
\hline & Outros & 17 & 9,69 \\
\hline & TOTAL & 174 & 100 \\
\hline Conforto & Dor & 47 & 53,41 \\
\hline \multirow{3}{*}{ Prejudicado } & Náuseas e vômitos & 7 & 7,96 \\
\hline & Outros & 34 & 38,63 \\
\hline & TOTAL & 88 & 100 \\
\hline \multirow[t]{2}{*}{ Nutrição } & Desconforto oral & 3 & 4,35 \\
\hline & Diminuição do desejo de comer & 30 & 43,48 \\
\hline \multirow[t]{5}{*}{ Corporais } & Náuseas e inapetência & 4 & 5,80 \\
\hline & Outros & 23 & 33,32 \\
\hline & Não informado & 6 & 8,70 \\
\hline & Náuseas e vômitos & 3 & 4,35 \\
\hline & TOTAL & 69 & $100 \%$ \\
\hline \multirow[t]{5}{*}{ Mobilidade física } & Dor & 7 & 19,45 \\
\hline & Câncer & 6 & 16,67 \\
\hline & Outros & 22 & 61,10 \\
\hline & Não informado & 1 & 2,78 \\
\hline & TOTAL & 36 & 100 \\
\hline \multirow[t]{3}{*}{ Risco para lesão } & Tendências hemorrágicas & 19 & 86,36 \\
\hline & Outros & 3 & 16,64 \\
\hline & TOTAL & 22 & $100 \%$ \\
\hline Sono e repouso & Dor & 12 & 57,14 \\
\hline \multirow{4}{*}{ Prejudicado } & Hospitalização & 4 & 19,05 \\
\hline & Outros & 4 & 19,05 \\
\hline & Não informado & 1 & 4,76 \\
\hline & TOTAL & 21 & $100 \%$ \\
\hline \multirow[t]{4}{*}{ Dor aguda } & Câncer & 7 & 33,33 \\
\hline & $\begin{array}{l}\text { Traumatismo tissular e espasmos da musculatura } \\
\text { reflexa }\end{array}$ & 5 & 23,81 \\
\hline & Outros & 9 & 42,86 \\
\hline & TOTAL & 21 & 100 \\
\hline
\end{tabular}

Fonte: DAME- Divisão de Arquivo Médico e Estatístico/ HOL, 2014.

A respeito das intervenções de enfermagem, nos anos de coleta do estudo, foram identificadas 1547 ações de enfermagem realizadas uma única 
vez ou diversas vezes durante o dia, no período dos dois anos do estudo. Dentre os cuidados de enfermagem as 14 principais intervenções são mostradas na tabela 3 abaixo:

TABELA 3 - Principais intervenções de enfermagem implementadas a criança e adolescente com câncer

\begin{tabular}{lcc}
\hline INTERVENÇÃO DE ENFERMAGEM & $\mathrm{N}$ & $\%$ \\
\hline Aferir Sinais Vitais & 211 & 13,64 \\
Controlar gotejamento & 209 & 13,51 \\
Observar venopunção & 191 & 12,35 \\
Acomodar no leito & 174 & 11,25 \\
Curativo de FO & 80 & 5,17 \\
Estimular aceitação da dieta & 76 & 4,91 \\
Orientar/ supervisionar cuidados higiênicos & 70 & 4,52 \\
Realizar PH urinário & 60 & 3,88 \\
Medir débito urinário & 56 & 3,62 \\
Utilização de cobertura para feridas & 35 & 2,26 \\
Avaliar dor & 35 & 2,26 \\
Curativo CVC & 34 & 2,20 \\
Bochecho para remissão de mucosite & 33 & 2,13 \\
Compressa bicarbonatada & 31 & 2,00 \\
\hline
\end{tabular}

Fonte: DAME - Divisão de Arquivo Médico e Estatístico/ HOL, 2014.

As intervenções de enfermagem foram em sua maioria para a aferição de sinais vitais, devido à grande labilidade do organismo da criança e adolescente com câncer. Além deste é possível visualizar a prevenção da lesão vascular a partir do controle de gotejamento venóclise e atenção aos sinais de flogose no acesso venoso periférico e central. Estas medidas são extremamente relevantes à criança e adolescente com câncer pelo fator da rápida evolução com neutropenia pelo tratamento e pela patologia oncológica e maior chance de infecção relacionada à assistência em saúde.

Além dos cuidados citados na tabela 3, foi possível identificar registro das seguintes atividades de enfermagem em oncologia pediátrica: Cuidados e auxílio na higiene, instalação de gel redutor de hematoma e edema, cuidados com gavagem, e observar evacuação (frequência e aspecto).

Quanto ao direcionamento dos cuidados de enfermagem identificados nos prontuários, segundo padronização do protocolo de pesquisa identificouse 308 relações dos cuidados de enfermagem com os sinais e sintomas descritos em prontuário é possível verificar que a concentração dos cuidados ocorreu 
aos sintomas de neutropenia $(\mathrm{n}=92)$, manejo das náuseas e vômitos $(\mathrm{n}=66)$, mucosite $(n=61)$ e manejo e controle da dor $(\mathrm{N}=35)$.

O câncer na criança, mais intensamente do que no adulto, determina expressões de pena e pesar, em razão do medo e mitos da doença oncológica. Para as crianças menores, o câncer pode estar relacionado a castigos por conduta inadequada. As privações do colo, do aconchego dos pais nos procedimentos de intervenção, causam grandes estresses para criança. O suporte emocional e a criatividade na arte do cuidar devem ser valorizados, requerendo habilidade técnica e empática (PARO; PARO; FERREIRA, 2005).

Em estudo realizado por na Colômbia em 2012 com 43 crianças sobre os principais diagnósticos de enfermagem em pacientes que recebem tratamento oncológico, verificou-se com maior frequência os seguintes títulos: Proteção Ineficaz 81,4\% ( $\mathrm{N}=35)$, Risco de infeç̧ão 79,1\% ( $\mathrm{N}=34)$, Risco de lesão $72,1 \%(\mathrm{~N}=31)$, Náusea $69,8 \%(\mathrm{~N}=30)$, Transtorno da imagem corporal 51,2\% (N=22), Fadiga 44,2\% ( $\mathrm{N}=19)$, Intolerância a atividade 39,5\% ( $\mathrm{N}=17)$, Déficit de atividades recreativas 39,5\% ( $\mathrm{N}=17)$, Dentição prejudicada 30,2\% ( $\mathrm{N}=13)$ e Temor 27,9\% ( $=13)$ (QUECHO, 2012).

Os diagnósticos deste estudo apresentam relação com a pesquisa. Salienta-se que no estudo, a taxonomia utilizada para o título diagnóstico é a NANDA II, e na instituição onde foi realizada a pesquisa deste estudo é empregado a taxonomia de Carpenito. No entanto, pode-se perceber que os diagnósticos de risco e que são relacionadas à imunossupressão e efeitos da quimioterapia antineoplásica são equivalentes, se comparados os dois estudos (QUECHO, 2012).

Outro fator bem evidente no ao final do estudo foram os casos de náuseas e vômitos que corresponderam a $(21,43 \%)$ relacionado a efeitos pós quimioterapia, causando um maior sofrimento aos pacientes. Náuseas e vômitos são manifestações frequentes nos pacientes com câncer e estão geralmente associados a quimioterapia, radioterapia, cirurgias e determinadas situações e circunstancias, assim é perfeitamente aceitável que um paciente com adequado controle sintomáticos de náuseas e vômitos, apresente uma melhor qualidade de vida, uma melhor aderência ao tratamento com menores custos e uma menor morbimortalidade e não necessite de eventuais reduções de dose das drogas, assim é fundamental que a enfermagem conheça não só as limitações do paciente mas também a fisiopatologia e uma adequada aplicação do arsenal farmacológico que irá auxiliar na melhora dos sintomas apresentados pelo paciente. O profissional da enfermagem tem subsídios suficientes para tratar e proporcionar uma melhor qualidade de vida ao paciente assim como uma melhor assistência, a partir da empoderamento do diagnóstico de enfermagem (SUTMOLLER, 2008).

Atualmente as patologias oncológicas infantojuvenis vêm representando um desafio para a equipe de saúde em especial a enfermagem fato evidente no aumento da formação de especialistas em enfermagem oncológica o que 
proporciona um grande avanço nos resultados de saúde e qualidade de vida dos pacientes infanto-juvenis. Já que o enfermeiro é o profissional de saúde responsável pelo cuidado direcionado ao paciente a partir de suas intervenções que visam controlar os efeitos negativos que surgem ao logo da doença durante o tratamento, não deixando de lado o apoio principal da família no processo de enfermagem, haja vista o aumento nos diagnósticos de enfermagem decorrentes da oncologia pediátrica (QUECHO, 2012).

\section{CONCLUSÃO}

Em relação à Sistematização da Assistência de Enfermagem (SAE) observou-se que os profissionais responsáveis por essa peculiaridade, em sua maioria estão empenhados em prestar uma assistência de qualidade a esses pacientes, encontramos em maioria dos prontuários de internação analisados a elaboração e aplicação da SAE para cada paciente atendido na clínica.

A melhoria da assistência de enfermagem faz-se necessário devido ao aumento da incidência de crianças com câncer que evoluem a óbito, e é neste sentido que a construção de um aporte teórico poderá contribuir na melhoria do atendimento de enfermagem e melhor qualidade de vida das crianças portadoras de câncer. Portanto esperamos que com este estudo possamos contribuir na qualidade e melhoria da assistência de enfermagem, fornecendo subsídios científicos para a prática do cuidar para o público infanto-juvenil acometido por câncer, alcançando a qualidade e segurança no processo de cuidar.

Não existiu nenhum conflito de interesse durante o planejamento, desenvolvimento ou análise dos dados deste estudo. A pesquisa foi realizada com financiamento próprio dos pesquisadores e faz parte de um recorte da monografia de graduação intitulada: "Perfil epidemiológico dos pacientes com câncer infanto-juvenil: Contribuições para o cuidado de enfermagem". Apresentada na Universidade Federal do Pará.

\section{REFERÊNCIAS}

AMADOR, Daniela Doulavince; GOMES, Isabelle Pimentel; COUTINHO, Simone Elisabeth Duarte; COSTA, Teresa Neumann Alcoforado; COLLET, Neusa. Concepção dos Enfermeiros acerca da Capacitação no cuidado à Criança com Câncer. Texto e contexto Enfermagem., Florianópolis, v. 20, n. 1, p. 94-101. Jan. 2011

Bastable, Susan B. O enfermeiro como educador: Princípios para o ensinoaprendizagem para a prática de enfermagem. $3^{\mathrm{a}}$ ed., São Paulo: Artmed; 2010.

LINS, Matheus R. A Criança com Câncer e a Família: Contexto, Descoberta e Ação. Cap.2 in Oncologia Pediátrica: Uma abordagem Multiprofissional. $1^{a}$ ed. São Paulo: Martinari; 2011. 
PARO, Daniela; PARO, Juliana; FERREIRA, Daise LM. O enfermeiro e o cuidar em oncologia pediátrica. Arq Ciênc Saúde., São José do Rio Preto, v.12, n.3, p.143-59. Julset. 2005.

POLIT, Denise F; BECK, Cheryl T, HUNGLER Bernadette P. Fundamentos de pesquisa em enfermagem: métodos, avaliação e utilização. $5^{\mathrm{a}}$ ed., Porto Alegre: Artmed; 2004.

QUECHO, Adrian. Prevalencia de los dianósicos de enfermeira em ninõs que reciben tratamento oncológico. Revista electrónica trimestral de enfermeira., Colômbia, v.27, n.1, p.77-86. Jan.2012

SILVA, Thiago Privado; LEITE, Jozéte Luzia; SANTOS, Nereida Lúcia Palko; SILVA, Ítalo Rodolfo; MENDONÇA, Ana Carolina Abeid; SANTOS, Maria José Carvalho; SILVA, Laura Johanson. Cuidados de Enfermagem à Criança com Câncer: Uma Revisão integrativa da Literatura. Revista de Enfermagem UFSM., Minas Gerais, v.3, n.1, p.68-78. Jan. 2013

SUTMOLLER, Christian. Náuseas e vômitos. In: Rotinas em Oncologia. Porto Alegre: Artmed; 2008. 